\title{
Exact and explicit solutions to the discrete nonlinear Schrödinger equation with a saturable nonlinearity
}

\author{
İsmail Aslan \\ Department of Mathematics, Izmir Institute of Technology, Urla, Izmir 35430, Turkey
}

\section{A R T I C L E I N F O}

\section{Article history:}

Received 9 August 2011

Received in revised form 4 October 2011

Accepted 6 October 2011

Available online 8 October 2011

Communicated by R. Wu

\section{Keywords:}

$\left(G^{\prime} / G\right)$-expansion method

Discrete nonlinear Schrödinger equation

Exact solutions

\begin{abstract}
A B S T R A C T
We analyze the discrete nonlinear Schrödinger equation with a saturable nonlinearity through the $\left(G^{\prime} / G\right)$-expansion method to present some improved results. Three types of analytic solutions with arbitrary parameters are constructed; hyperbolic, trigonometric, and rational which have not been explicitly computed before.
\end{abstract}

(C) 2011 Elsevier B.V. All rights reserved.

\section{Introduction}

Nonlinear differential-difference equations (NDDEs) or lattice equations arise frequently in the mathematical analysis of a wide variety of problems in applied physical and mathematical sciences. It is becoming even more desirable for scientists to pursue study and research on NDDEs. Their crucial role has encouraged mathematicians, physicists, and other scientists to develop many integrable NDDEs. To make mention of a few; the Volterra lattice equation [1], the Ablowitz-Ladik lattice equation [2], the Toda lattice equation [3], the discrete KdV equation [4], the discrete sineGordon equation [5], and so forth. Whereas difference equations are being fully discretized, NDDEs are semi-discretized with some (or all) of their space variables discretized while time is usually kept continuous. Hence, they can be considered as hybrid systems. In recent years, considerable attention has been given to the problem of exactly solving NDDEs. Some authors [6-10] put forth new modifications of the existing methods to tackle NDDEs. Moreover, Ma and You [11] used Casoratian technique for constructing rational solutions to the Toda lattice equation. Dai and Zhang [12] utilized the extended tanh-function approach to obtain various solutions for the discrete complex dissipative cubic-quintic Ginzburg-Landau equation with non-local quintic term. Using a multiscale expansion method, Syafwan et al. [13] reduced a particular type of parametrically driven discrete Klein-Gordon system to a discrete nonlinear Schrödinger equation. Kou et al. [14] modified the bilinear Bäcklund transformation for the discrete sine-Gordon equation to derive variety of solutions. However, the methods com-

E-mail address: ismailaslan@iyte.edu.tr. monly used are usually restricted and cannot be used for numerous realistic scenarios.

The discrete nonlinear Schrödinger equation (DNSE) is one of the most fundamental nonlinear lattice models [2]. It arises in nonlinear optics as a model of infinite wave guide arrays [15] and has been recently implemented to describe Bose-Einstein condensates in optical lattices [16]. The class of DNSE models with saturable nonlinearity is also of particular interest in their own right, due to a feature first unveiled in [17]. In this study, we focus on the so-called DNSE with a saturable nonlinearity $[18,19]$

$i \frac{\partial \psi_{n}}{\partial t}+\left(\psi_{n+1}+\psi_{n-1}-2 \psi_{n}\right)+\frac{v\left|\psi_{n}\right|^{2}}{1+\mu\left|\psi_{n}\right|^{2}} \psi_{n}=0$

which describes optical pulse propagation in various doped fibres; $\psi_{n}$ is a complex valued wave function at site $n$, while $\nu$ and $\mu$ are real parameters. This equation admits the Hamiltonian system

$H=\sum_{n=1}^{N}\left[\left|\psi_{n}-\psi_{n+1}\right|^{2}-\frac{v}{\mu}\left|\psi_{n}\right|^{2}+\frac{v}{\mu^{2}} \ln \left(1+\mu\left|\psi_{n}\right|^{2}\right)\right]$,

such that Eq. (1) is given by $i \psi_{n}=\partial H / \partial \psi_{n}^{*}$.

Nowadays, some modern analytic methods are available for analyzing nonlinear evolution-type equations. For instance, tanh function method [20], homotopy perturbation method [21], variational iteration method [22], first integral method [23], Expfunction method [24], multiple Exp-function method [25], linear superposition principle [26], Wronskian technique [27], the $F$ expansion method [28], the Jacobi elliptic-function method [29], the similarity transformation method [30], the ansätze method [31]. On the other hand, since it was first enunciated in 2008 by 
Wang et al. [32], the $\left(G^{\prime} / G\right)$-expansion method was very well received and a number of studies refining the initial idea appeared in the research literature [33-35]. The basic idea of the $\left(G^{\prime} / G\right)$ expansion method lies in the fact that it takes full advantage of linear theory. Being straightforward and concise, we can find some publications in which the method has been adapted, generalized and extended for various kinds of nonlinear problems [36-41]. Hence, the $\left(G^{\prime} / G\right)$-expansion method provides a valuable addition to the wave theory. We think that the application of new methods to the DNSE with a saturable nonlinearity (1) is still an interesting and important problem of mathematical physics. In the present Letter, we aim to derive some further results for Eq. (1) using the $\left(G^{\prime} / G\right)$-expansion method.

The outline of the Letter is as follows: In Section 2, we present our methodology. In Section 3, we study our problem. We end our analysis with a conclusion in Section 4.

\section{The $\left(G^{\prime} / G\right)$-expansion method for NDDEs}

Before proceeding, let us consider a system of $M$ polynomial NDDEs of the form

$$
\begin{aligned}
& P\left(\mathbf{u}_{\mathbf{n}+\mathbf{p}_{1}}(\mathbf{x}), \ldots, \mathbf{u}_{\mathbf{n}+\mathbf{p}_{k}}(\mathbf{x}), \ldots, \mathbf{u}_{\mathbf{n}+\mathbf{p}_{1}}^{\prime}(\mathbf{x}), \ldots, \mathbf{u}_{\mathbf{n}+\mathbf{p}_{k}}^{\prime}(\mathbf{x}), \ldots,\right. \\
& \left.\mathbf{u}_{\mathbf{n}+\mathbf{p}_{1}}^{(r)}(\mathbf{x}), \ldots, \mathbf{u}_{\mathbf{n}+\mathbf{p}_{k}}^{(r)}(\mathbf{x})\right)=0,
\end{aligned}
$$

in which the dependent variable $\mathbf{u}_{\mathbf{n}}$ have $M$ components $u_{i, \mathbf{n}}$ and so do its shifts, the continuous variable $\mathbf{x}$ has $N$ components $x_{i}$, the discrete variable $\mathbf{n}$ has $Q$ components $n_{j}$, the $k$ shift vectors $\mathbf{p}_{i} \in$ $\mathbb{Z}^{Q}$ and $\mathbf{u}^{(r)}(\mathbf{x})$ denotes the collection of mixed derivative terms of order $r$. To solve Eq. (3), we first make the transformation

$$
\begin{aligned}
& \mathbf{u}_{\mathbf{n}+\mathbf{p}_{s}}(\mathbf{x})=\mathbf{U}_{\mathbf{n}+\mathbf{p}_{s}}\left(\xi_{\mathbf{n}}\right), \\
& \xi_{\mathbf{n}}=\sum_{i=1}^{Q} d_{i} n_{i}+\sum_{j=1}^{N} c_{j} x_{j}+\zeta \quad(s=1,2, \ldots, k),
\end{aligned}
$$

where the coefficients $c_{1}, c_{2}, \ldots, c_{N}, d_{1}, d_{2}, \ldots, d_{Q}$ and the phase $\zeta$ are all constants. Then, Eq. (3) can be reduced to

$$
\begin{gathered}
\Delta\left(\mathbf{U}_{\mathbf{n}+\mathbf{p}_{1}}\left(\xi_{\mathbf{n}}\right), \ldots, \mathbf{U}_{\mathbf{n}+\mathbf{p}_{k}}\left(\xi_{\mathbf{n}}\right), \ldots, \mathbf{U}_{\mathbf{n}+\mathbf{p}_{1}}^{\prime}\left(\xi_{\mathbf{n}}\right), \ldots, \mathbf{U}_{\mathbf{n}+\mathbf{p}_{k}}^{\prime}\left(\xi_{\mathbf{n}}\right),\right. \\
\left.\ldots, \mathbf{U}_{\mathbf{n}+\mathbf{p}_{1}}^{(r)}\left(\xi_{\mathbf{n}}\right), \ldots, \mathbf{U}_{\mathbf{n}+\mathbf{p}_{k}}^{(r)}\left(\xi_{\mathbf{n}}\right)\right)=0 .
\end{gathered}
$$

We assume that Eq. (5) admits a solution in the finite series expansion form

$$
\mathbf{U}_{\mathbf{n}}\left(\xi_{\mathbf{n}}\right)=\sum_{l=0}^{m} a_{l}\left(\frac{G^{\prime}\left(\xi_{\mathbf{n}}\right)}{G\left(\xi_{\mathbf{n}}\right)}\right)^{l}, \quad a_{m} \neq 0,
$$

where the positive integer $m$ is determined by balancing the highest-order nonlinear term(s) and the highest-order derivative term(s) in Eq. (5), $a_{l}$ 's are constants to be specified at the stage of solving Eq. (5), and $G\left(\xi_{\mathbf{n}}\right)$ is the general solution of the secondorder linear equation

$G^{\prime \prime}\left(\xi_{\mathbf{n}}\right)+k G\left(\xi_{\mathbf{n}}\right)=0$,

where $k$ is an arbitrary constant and the prime denotes the derivative with respect to $\xi_{\mathbf{n}}$.

Lemma. The following expressions hold:

$\frac{G^{\prime}\left(\xi_{\mathbf{n}}\right)}{G\left(\xi_{\mathbf{n}}\right)}=\sqrt{-k}\left(\frac{C_{1} \cosh \left(\sqrt{-k} \xi_{\mathbf{n}}\right)+C_{2} \sinh \left(\sqrt{-k} \xi_{\mathbf{n}}\right)}{C_{1} \sinh \left(\sqrt{-k} \xi_{\mathbf{n}}\right)+C_{2} \cosh \left(\sqrt{-k} \xi_{\mathbf{n}}\right)}\right), \quad k<0$,

$\frac{G^{\prime}\left(\xi_{\mathbf{n}}\right)}{G\left(\xi_{\mathbf{n}}\right)}=\sqrt{k}\left(\frac{-C_{1} \sin \left(\sqrt{k} \xi_{\mathbf{n}}\right)+C_{2} \cos \left(\sqrt{k} \xi_{\mathbf{n}}\right)}{C_{1} \cos \left(\sqrt{k} \xi_{\mathbf{n}}\right)+C_{2} \sin \left(\sqrt{k} \xi_{\mathbf{n}}\right)}\right), \quad k>0$,
$\frac{G^{\prime}\left(\xi_{\mathbf{n}}\right)}{G\left(\xi_{\mathbf{n}}\right)}=\frac{C_{1}}{C_{1} \xi_{\mathbf{n}}+C_{2}}, \quad k=0$,

where $C_{1}$ and $C_{2}$ are arbitrary constants.

Proof. From the linear theory, the general solution of Eq. (7) is well known to us.

Proposition. The following shift formulas hold:

$$
\begin{aligned}
& \mathbf{U}_{\mathbf{n}+\mathbf{p}_{s}}\left(\xi_{\mathbf{n}}\right)=\sum_{l=0}^{m} a_{l}\left(\frac{\sqrt{-k} G^{\prime}\left(\xi_{\mathbf{n}}\right)-k \tanh \left(\sqrt{-k} \varphi_{s}\right) G\left(\xi_{\mathbf{n}}\right)}{\sqrt{-k} G\left(\xi_{\mathbf{n}}\right)+\tanh \left(\sqrt{-k} \varphi_{s}\right) G^{\prime}\left(\xi_{\mathbf{n}}\right)}\right)^{l}, \\
& a_{m} \neq 0, \quad k<0, \mathbf{U}_{\mathbf{n}+\mathbf{p}_{s}}\left(\xi_{\mathbf{n}}\right)=\sum_{l=0}^{m} a_{l}\left(\frac{\sqrt{k} G^{\prime}\left(\xi_{\mathbf{n}}\right)-k \tan \left(\sqrt{k} \varphi_{s}\right) G\left(\xi_{\mathbf{n}}\right)}{\sqrt{k} G\left(\xi_{\mathbf{n}}\right)+\tan \left(\sqrt{k} \varphi_{s}\right) G^{\prime}\left(\xi_{\mathbf{n}}\right)}\right)^{l}, \\
& a_{m} \neq 0, \quad k>0, \\
& \mathbf{U}_{\mathbf{n}+\mathbf{p}_{s}}\left(\xi_{\mathbf{n}}\right)=\sum_{l=0}^{m} a_{l}\left(\frac{G^{\prime}\left(\xi_{\mathbf{n}}\right)}{G\left(\xi_{\mathbf{n}}\right)+\varphi_{s} G^{\prime}\left(\xi_{\mathbf{n}}\right)}\right)^{l}, \\
& a_{m} \neq 0, \quad k=0 .
\end{aligned}
$$

Proof. By a straightforward calculation, we can get the identity

$\xi_{\mathbf{n}+\mathbf{p}_{s}}=\xi_{\mathbf{n}}+\varphi_{s}, \quad \varphi_{s}=p_{s 1} d_{1}+p_{s 2} d_{2}+\cdots+p_{s Q} d_{Q}$

where $p_{s j}$ is the $j$ th component of the shift vector $\mathbf{p}_{s}$. Taking the trigonometric/hyperbolic function identities into account and using Lemma along with (10) reveal (9a)-(9c).

Thus, substituting (6) and (9a)-(9c) together with (7) into Eq. (5), equating the coefficients of $\left(G^{\prime}\left(\xi_{\mathbf{n}}\right) / G\left(\xi_{\mathbf{n}}\right)\right)^{l}(l=0,1,2, \ldots)$ to zero, we obtain a system of nonlinear algebraic equations from which the undetermined constants $a_{l}, d_{i}, c_{j}$, and $k$ can be explicitly found (probably, with the help of a symbolic computation system).

Remark 1. The basic $\left(G^{\prime} / G\right)$-expansion method takes the auxiliary equation $G^{\prime \prime}+q G^{\prime}+k G=0$ into account where $q$ and $k$ are arbitrary constants. Recently, Aslan [42] showed that the parameter $q$ is superfluous, namely, one can assume $q=0$ without loss of generality. This modification allows us to minimize the number of the parameters at the outset without affecting the generality of the solutions.

\section{Analysis of Eq. (1)}

In order to tackle Eq. (1), we first make the transformation

$\psi_{n}=\varphi_{n}\left(\xi_{n}\right) e^{-i(\sigma t+\rho)}, \quad \xi_{n}=\alpha n+\beta$,

where $\sigma$ and $\alpha$ are real parameters to be determined, while $\rho$ and $\beta$ are arbitrary real constants. The substitution of (11) into Eq. (1) leads to the nonlinear difference equation

$(\sigma-2) \varphi_{n}\left(\xi_{n}\right)+\varphi_{n+1}\left(\xi_{n+1}\right)+\varphi_{n-1}\left(\xi_{n-1}\right)+\frac{\nu \varphi_{n}^{3}\left(\xi_{n}\right)}{1+\mu \varphi_{n}^{2}\left(\xi_{n}\right)}=0$.

According to the $\left(G^{\prime} / G\right)$-expansion method, we assume that Eq. (12) assumes a solution of the form

$\varphi_{n}\left(\xi_{n}\right)=a_{0}+a_{1}\left(\frac{G^{\prime}\left(\xi_{n}\right)}{G\left(\xi_{n}\right)}\right), \quad a_{1} \neq 0$,

where $G\left(\xi_{n}\right)$ satisfies Eq. (7), while $a_{0}$ and $a_{1}$ are arbitrary constants to be determined. 


\subsection{The solution functions of hyperbolic type}

In the case $k<0$, we first derive the expressions $\varphi_{n \pm 1}\left(\xi_{n \pm 1}\right)$ in accordance with (9a) and substitute them along with (13) into Eq. (12). Then, clearing the denominator and setting the coefficients of $\left(G^{\prime} / G\right)^{l}(0 \leqslant l \leqslant 5)$ to zero, we derive a system of nonlinear algebraic equations for $a_{0}, a_{1}, \sigma, \rho, \alpha, \beta, v, \mu$, and $k$. Solving the resultant system, we get the following solution set

$$
\begin{gathered}
\left\{v=2 \mu \operatorname{sech}^{2}(\sqrt{-k} \alpha), \sigma=2 \tanh ^{2}(\sqrt{-k} \alpha),\right. \\
\left.a_{1}= \pm \tanh (\sqrt{-k} \alpha) / \sqrt{-k} \sqrt{-\mu}, a_{0}=0\right\},
\end{gathered}
$$

and the corresponding discrete hyperbolic functions solution to Eq. (1) as

$$
\begin{aligned}
\psi_{n, 1}^{ \pm}(t)= & \pm \frac{\tanh (\sqrt{-k} \alpha)}{\sqrt{-\mu}} \\
& \times\left(\frac{C_{1} \cosh (\sqrt{-k}(\alpha n+\beta))+C_{2} \sinh (\sqrt{-k}(\alpha n+\beta))}{C_{1} \sinh (\sqrt{-k}(\alpha n+\beta))+C_{2} \cosh (\sqrt{-k}(\alpha n+\beta))}\right) \\
& \times \exp \left(-i\left(2 \tanh ^{2}(\sqrt{-k} \alpha) t+\rho\right)\right),
\end{aligned}
$$

where the parameters satisfy $v=2 \mu \operatorname{sech}^{2}(\sqrt{-k} \alpha)$ and $\mu<0$, while $\alpha, \beta, \rho, k(<0), C_{1}, C_{2}$ remain arbitrary.

\subsection{The solution functions of trigonometric type}

In the case $k>0$, we first derive the expressions $\varphi_{n \pm 1}\left(\xi_{n \pm 1}\right)$ in accordance with (9b) and substitute them along with (13) into Eq. (12). Then, clearing the denominator and setting the coefficients of $\left(G^{\prime} / G\right)^{l}(0 \leqslant l \leqslant 5)$ to zero, we derive a system of nonlinear algebraic equations for $a_{0}, a_{1}, \sigma, \alpha, v, \mu$, and $k$. Solving the resultant system, we get the following solution sets

$$
\begin{gathered}
\left\{v=2 \mu \sec ^{2}(\sqrt{k} \alpha), \sigma=-2 \tan ^{2}(\sqrt{k} \alpha),\right. \\
\left.a_{1}= \pm \tan (\sqrt{k} \alpha) / \sqrt{k} \sqrt{-\mu}, a_{0}=0\right\},
\end{gathered}
$$

and the corresponding discrete trigonometric function solutions to Eq. (1) as

$$
\begin{aligned}
\psi_{n, 2}^{ \pm}(t)= & \pm \frac{\tan (\sqrt{k} \alpha)}{\sqrt{-\mu}} \\
& \times\left(\frac{-C_{1} \sin (\sqrt{k}(\alpha n+\beta))+C_{2} \cos (\sqrt{k}(\alpha n+\beta))}{C_{1} \cos (\sqrt{k}(\alpha n+\beta))+C_{2} \sin (\sqrt{k}(\alpha n+\beta))}\right) \\
& \times \exp \left(-i\left(-2 \tan ^{2}(\sqrt{k} \alpha) t+\rho\right)\right)
\end{aligned}
$$

where the parameters satisfy $\nu=2 \mu \sec ^{2}(\sqrt{k} \alpha)$ and $\mu<0$, while $\alpha, \beta, \rho, k(>0), C_{1}, C_{2}$ remain arbitrary.

\subsection{The solution functions of rational type}

In the case $k=0$, we first derive the expressions $\varphi_{n \pm 1}\left(\xi_{n \pm 1}\right)$ in accordance with (9c) and substitute them along with (13) into Eq. (12). Then, clearing the denominator and setting the coefficients of $\left(G^{\prime} / G\right)^{l}(0 \leqslant l \leqslant 5)$ to zero, we derive a system of nonlinear algebraic equations for $a_{0}, a_{1}, \sigma, \alpha, v$, and $\mu$. Solving the resultant system, we get the following solution sets

$\left\{a_{1}= \pm \frac{\alpha}{\sqrt{-\mu}}, v=2 \mu, \sigma=0, a_{0}=0\right\}$

and the corresponding discrete rational function solutions to Eq. (1) as

$\psi_{n, 3}^{ \pm}(t)= \pm \frac{\alpha C_{1}}{\sqrt{-\mu}\left(C_{1}(\alpha n+\beta)+C_{2}\right)} \times \exp (-i \rho)$,

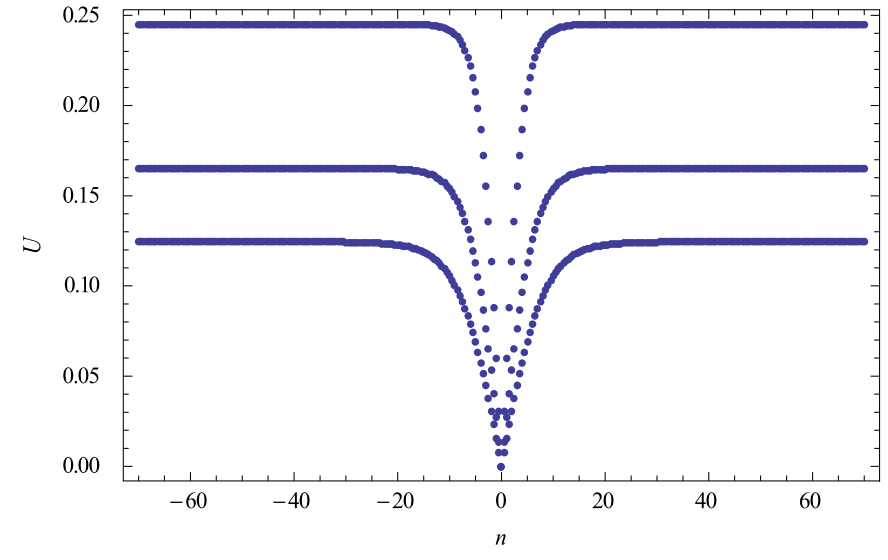

Fig. 1.

where the parameters satisfy $v=2 \mu$ and $\mu<0$, while $\alpha, \beta, \rho, C_{1}$, and $C_{2}$ remain arbitrary. It is worth to remark that the functions (19) are non-constant steady-state rational solutions to Eq. (1) and they have not been obtained before.

\subsection{The solution functions of special types}

By assigning special values to the arbitrary parameters in the expressions (15) and (17), it is possible to derive some specific exact solutions as follows:

As a first example, if we let " $C_{1}=0$ and $C_{2} \neq 0$ " or " $C_{1} \neq 0$ and $C_{2}=0$ " in (15), respectively, then we get formal solitary wave solutions to Eq. (1) as

$$
\begin{aligned}
\psi_{n, 4}^{ \pm}(t)= & \pm \frac{\tanh (\sqrt{-k} \alpha)}{\sqrt{-\mu}} \tanh (\sqrt{-k}(\alpha n+\beta)) \\
& \times \exp \left(-i\left(2 \tanh ^{2}(\sqrt{-k} \alpha) t+\rho\right)\right), \\
\psi_{n, 5}^{ \pm}(t)= & \pm \frac{\tanh (\sqrt{-k} \alpha)}{\sqrt{-\mu}} \operatorname{coth}(\sqrt{-k}(\alpha n+\beta)) \\
& \times \exp \left(-i\left(2 \tanh ^{2}(\sqrt{-k} \alpha) t+\rho\right)\right),
\end{aligned}
$$

where $\nu=2 \mu \operatorname{sech}^{2}(\sqrt{-k} \alpha)$ and $\mu<0$, while $\alpha, \beta, \rho$, and $k(<0)$ remain arbitrary.

As a second example, if we let " $C_{1} \neq 0$ and $C_{2}=0$ " or " $C_{1}=0$ and $C_{2} \neq 0$ " in (17) respectively, then we get formal periodic wave solutions to Eq. (1) as

$$
\begin{aligned}
\psi_{n, 6}^{\mp}(t)= & \mp \frac{\tan (\sqrt{k} \alpha)}{\sqrt{-\mu}} \tan (\sqrt{k}(\alpha n+\beta)) \\
& \times \exp \left(-i\left(-2 \tan ^{2}(\sqrt{k} \alpha) t+\rho\right)\right), \\
\psi_{n, 7}^{ \pm}(t)= & \pm \frac{\tan (\sqrt{k} \alpha)}{\sqrt{-\mu}} \cot (\sqrt{k}(\alpha n+\beta)) \\
& \times \exp \left(-i\left(-2 \tan ^{2}(\sqrt{k} \alpha) t+\rho\right)\right),
\end{aligned}
$$

where $v=2 \mu \sec ^{2}(\sqrt{k} \alpha)$ and $\mu<0$, while $\alpha, \beta, \rho$, and $k(>0)$ remain arbitrary.

Remark 2. Soliton solutions are of physical importance. We have obtained abundant exact traveling wave solutions for Eq. (1). To analyze their properties, we consider the solutions (20) and (21) only for simplicity. Fig. 1 shows the profile of the modulus $U \equiv$ $\left|\psi_{n, 4}^{ \pm}(t)\right|$ of the dark solitary wave solution (20) for the parameters involved $k=\mu=-1, \rho=\beta=0, \alpha=0.4,0.6,0.8$. Fig. 2 displays the profile of the modulus $U \equiv\left|\psi_{n, 5}^{ \pm}(t)\right|$ of the exotic wave solution (21) for the parameters $k=\mu=-1, \rho=\beta=0, \alpha=0.4$. 


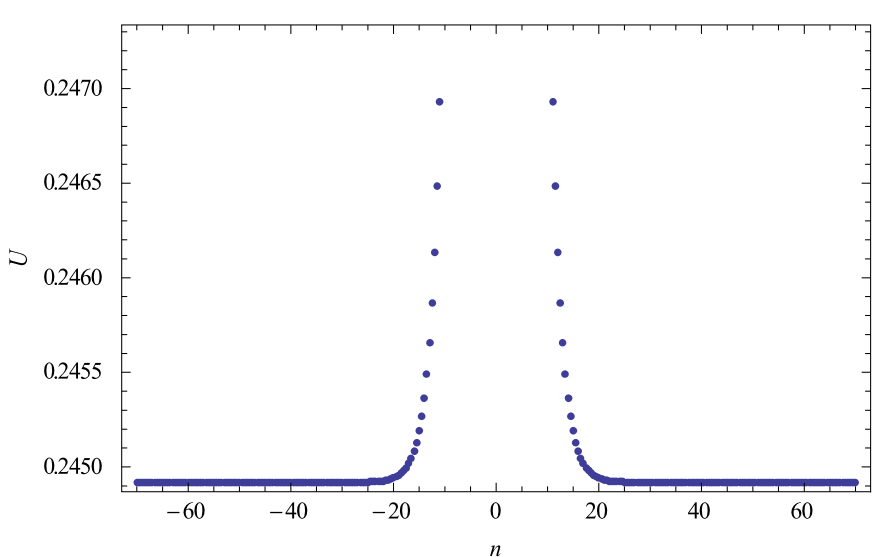

Fig. 2.

It is worthwhile to mention here that we could not give further details about the real physical meaning of our exact solutions because of the lack of theoretical and experimental basis related to the obtained solutions. It is hoped that the experimental outcomes observed here can be realized in some fields in the future. Though the solutions provided here constitute only a small part of a large variety of possible solutions for the equation discussed, they might serve as seeding solutions for a class of localized structures which exist in this equation. Of course, the linear stability analysis of exact solutions, as presented in [43], is of experimental interest. This problem will be under investigation for another Letter.

Remark 3. It seems that the multiple Exp-function method [25] is more general than the $\left(G^{\prime} / G\right)$-expansion method. The adopted $\left(G^{\prime} / G\right)$-expansion method actually uses the same idea as the one using an integrable ODE $v_{\xi}=a v+b v^{2}$ with constants $a$ and $b$, which was presented for the first time in [31]. Recently, the $\left(G^{\prime} / G\right)$-expansion method has also attracted some comments [44$46]$ in which it is claimed that $\left(G^{\prime} / G\right)$-expansion method is equivalent to the extended tanh function method (or the Ricatti subequation method) [47]. However, we believe that one should always have the freedom of choosing one of the equivalent methods for analyzing a new equation or a previously unstudied (or partially studied) problem.

\section{Conclusion}

We systematically investigated the DNSE with a saturable nonlinearity, which is a very crucial model for the applied physical and mathematical sciences, for analytic solutions using one of the most recent methods, namely, the $\left(G^{\prime} / G\right)$-expansion method. We confirmed that the model supports three types of solutions with arbitrary parameters; hyperbolic, trigonometric, and rational. It ap- pears that some of the previously known results are particular cases of ours. The rational solutions are computed here for the first time and they could not be obtained by other methods.

\section{Acknowledgements}

The author thanks the anonymous referees for the valuable and useful suggestions.

\section{References}

[1] M. Wadati, Progr. Theoret. Phys. Suppl. 59 (1976) 36.

[2] M.J. Ablowitz, J. Ladik, J. Math. Phys. 16 (1975) 598.

[3] M. Toda, Theory of Nonlinear Lattices, Springer, 1989.

[4] Y. Ohta, R. Hirota, J. Phys. Soc. Jpn. 60 (1991) 2095.

[5] S.J. Orfandis, Phys. Rev. D 18 (1978) 3828.

[6] S.D. Zhu, Int. J. Nonlinear Sci. Numer. Simul. 8 (2007) 461.

[7] S.D. Zhu, Y.M. Chu, S.L. Qiu, Comput. Math. Appl. 58 (2009) 2398

[8] P. Yang, Y. Chen, Z.B. Li, Appl. Math. Comput. 210 (2009) 362

[9] Z. Wang, W.X. Ma, Math. Methods Appl. Sci. 33 (2010) 1463.

[10] C.Q. Dai, Y.Y. Wang, Phys. Scr. 78 (2008) 015013 (6 pp.).

[11] W.X. Ma, Y. You, Chaos Solitons Fractals 22 (2004) 395.

[12] C. Dai, J. Zhang, Opt. Commun. 263 (2006) 309.

[13] M. Syafwan, H. Susanto, S.M. Cox, Phys. Rev. E 81 (2010) 026207.

[14] X. Kou, D.J. Zhang, Y. Shi, S.L. Zhao, Commun. Theor. Phys. 55 (2011) 545.

[15] H.S. Eisenberg, Y. Silberberg, R. Morandotti, A.R. Boyd, J.S. Aitchison, Phys. Rev. Lett. 81 (1998) 3383.

[16] A. Trombettoni, A. Smerzi, Phys. Rev. Lett. 86 (2001) 2353.

[17] L. Hadžievski, A. Maluckov, M. Stepić, D. Kip, Phys. Rev. Lett. 93 (2004) 033901.

[18] S. Gatz, J. Herrmann, J. Opt. Soc. Amer. B 8 (1991) 2296.

[19] S. Gatz, J. Herrmann, Opt. Lett. 17 (1992) 4846.

[20] W. Malfliet, W. Hereman, Phys. Scr. 54 (1996) 563.

[21] J.H. He, Commun. Nonlinear Sci. Numer. Simul. 3 (1998) 92.

[22] J.H. He, Commun. Nonlinear Sci. Numer. Simul. 2 (1997) 230

[23] Z.S. Feng, J. Phys. A: Math. Gen. 35 (2002) 343.

[24] J.H. He, X.H. Wu, Chaos Solitons Fractals 30 (2006) 700.

[25] W.X. Ma, T. Huang, Y. Zhang, Phys. Scr. 82 (2010) 065003.

[26] W.X. Ma, E. Fan, Comput. Math. Appl. 61 (2011) 950.

[27] W.X. Ma, Y. You, Trans. Amer. Math. Soc. 357 (2004) 1753.

[28] J.F. Zhang, C.Q. Dai, Q. Yang, J.M. Zhu, Opt. Commun. 252 (2005) 408

[29] C.Q. Dai, J.F. Zhang, Internat. J. Modern Phys. B 19 (2005) 2129.

[30] C.Q. Dai, Y.Y. Wang, J.F. Zhang, Opt. Lett. 35 (2010) 1437.

[31] W.X. Ma, B. Fuchssteiner, Int. J. Non-Linear Mech. 31 (1996) 329.

[32] M. Wang, X. Li, J. Zhang, Phys. Lett. A 372 (2008) 417.

[33] İ. Aslan, T. Öziș, Appl. Math. Comput. 209 (2009) 425.

[34] T. Öziş, İ. Aslan, Z. Naturforsch. 64a (2009) 15.

[35] T. Öziş, İ. Aslan, Commun. Theor. Phys. 51 (2009) 577.

[36] S. Zhang, J.L. Tong, W. Wang, Phys. Lett. A 372 (2008) 2254.

[37] S. Zhang, L. Dong, J. Ba, Y. Sun, Phys. Lett. A 373 (2009) 905.

[38] İ. Aslan, Appl. Math. Comput. 215 (2009) 3140.

[39] İ. Aslan, J. Phys. A: Math. Theor. 43 (2010) 395207.

[40] İ. Aslan, Canad. J. Phys. 89 (2011) 857.

[41] B. Tang, Y. He, L. Wei, S. Wang, Phys. Lett. A 375 (2011) 3355.

[42] I. Aslan, Appl. Math. Comput. 217 (2010) 937.

[43] C.Q. Dai, R.P. Chen, G.Q. Zhou, J. Phys. B: At. Mol. Opt. Phys. 44 (2011) 145401.

[44] C.P. Liu, Commun. Theor. Phys. 51 (2009) 985.

[45] Y.L. Zhao, Y.P. Liu, Z.B. Li, Chin. Phys. B 19 (2010) 030306.

[46] P. Zhu, Commun. Theor. Phys. 52 (2009) 206

[47] E.G. Fan, Phys. Lett. A 277 (2000) 212. 